\title{
Erratum to: The advantages of tomosynthesis for evaluating bisphosphonate-related atypical femur fractures compared to radiography
}

\author{
Andrew Petraszko ${ }^{1} \cdot$ Daniel Siegal $^{1} \cdot$ Michael Flynn $^{1} \cdot$ Sudhaker D. Rao ${ }^{2}$. \\ Ed Peterson $^{3} \cdot$ Marnix van Holsbeeck $^{1}$
}

Published online: 22 February 2016

(C) ISS 2016

Erratum to: Skeletal Radiol

10.1007/s00256-016-2342-6

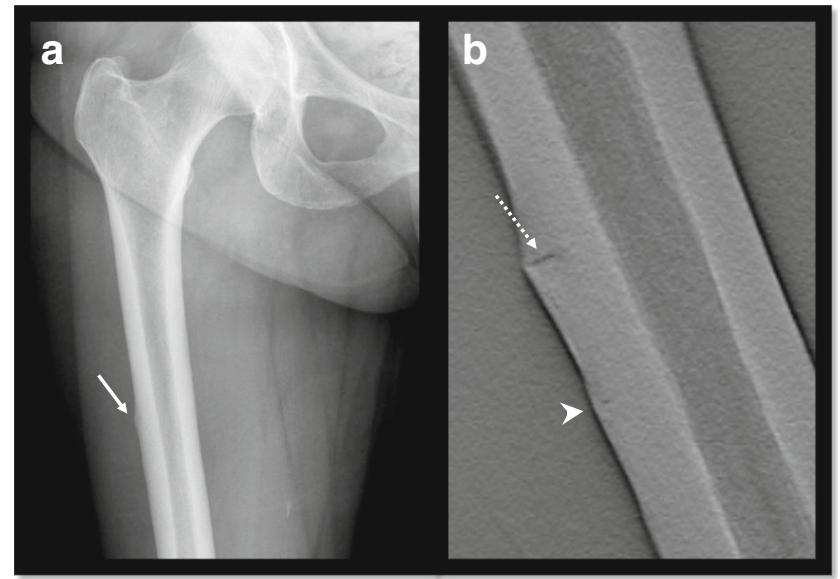

Fig. 2 A 75 year-old female with a 7 year history of bisphosphonate use. Only a single cortical beak (arrow) was seen on anteroposterior (AP) radiographs (a). In addition to showing the BP-AFF corresponding to the radiograph (dashed arrow), the AP tomosynthesis revealed another unsuspected BP-AFF line (arrowhead) more inferiorly not associated with cortical beaking (b). Thus, BP-AFF may exist in the total absence of any radiographic signs

Michael Flynn

mikef@rad.hfh.edu

Sudhaker D. Rao

Srao1@hfhs.org

Ed Peterson

Epeters1@hfhs.org

Marnix van Holsbeeck marnix@rad.hfh.edu

1 Department of Radiology, Henry Ford Hospital, 2799 West Grand Blvd, Detroit, MI 48202, USA

2 Division of Endocrinology, and Bone \& Mineral Research Laboratory, Henry Ford Hospital, 2799 West Grand Blvd, Detroit, MI 48202, USA

3 Department of Public Health Sciences, Office 3C, 1 Ford Place, Detroit, MI 48202, USA 